\title{
Fuzzy Control of Yaw and Roll Anglesof a Simulated Helicopter Model Includes Articulated Manipulators
}

\author{
Hossein Sadegh Lafmejani ${ }^{{ }^{1}}$, Hassan Zarabadipour ${ }^{2}$ \\ Faculty of Technical and Engineering, Imam Khomeini International University, \\ Qazvin, Iran, Tel: +98 (281) 8371753 \\ *Corresponding author, e-mail: h_sadegh@ikiu.ac.ir ${ }^{1}$, hassan.zarabadipour@gmail.com²
}

\begin{abstract}
Fuzzy logic controller (FLC) is a heuristic method by If-Then Rules which resembles human intelligence and it is a good method for designing Non-linear control systems. In this paper, an arbitrary helicopter model includes articulated manipulators has been simulated with Matlab SimMechanics toolbox. Due to the difficulties of modeling this complex system, a fuzzy controller with simple fuzzy rules has been designed for its yaw and roll angles in order to stabilize the helicopter while it is in the presence of disturbances orits manipulators are moving for a task. Results reveal that a simple FLC can appropriatelycontrol this system.
\end{abstract}

Keywords: fuzzy logic controller, helicopter, roll, simmechanics toolbox, yaw

\section{Introduction}

Helicopter is a vehicle with high weight and it is designed and made in different types and shapes and for variety of applications like industry, investigation, transportation and many other usages. In other word, helicopter is a multitasking system that is very hard to be controlled in the presence of disturbances and uncertainties. So intelligence control is a very good method for controlling the operation of this system.

There are so many papers about controlling of helicopterslike [1-7], but as I know, there is no paper about controlling of a helicopter includes manipulators in front of it. In order to study a helicopter with this characteristic, it should be simulated and Matlab SimMechanics toolbox is a good software for doing that as it is described briefly in [8].

As we mentioned above, modeling systems like helicopter is troublesome and sometimes impossible using the laws of physics. Therefore, it is not suitable to use classical controllers for these complex nonlinear control applications. Fuzzy logic controller is a suitable, useful and heuristic method for the control of complicated processes in presence of disturbances and uncertainties. In this method there is no need of system modeling or complex mathematical equations governing from the relationship between inputs and outputs. In fuzzy logic we use fuzzy rules easily, even by non- experts. In fuzzy logic the behavior of the system is characterized using human knowledge which leads to the design of control algorithm on the basis of fuzzy rules. As a result, fuzzy logic controller delivers a better performance in cases where the conventional controller does not cope well with non-linearity of a process under control [9]. There are so many papers published using the fuzzy logic controller for controlling the desired systems $[1,5],[10-16]$. So, in this paper we used fuzzy logic controller in order to control the yaw and roll angles of an arbitrary helicopter includes manipulators simulated with Matlab SimMechanics toolbox.

\section{System Description}

The system has been considered in this paper is a helicopter model that have two 2degree of freedom manipulators in the front of it. This system is shown in Figure 1 from designing in SimMechanics toolbox. These two manipulators are used for a specific task that is not important for us in this paper. The theory behind this system is shown in Figure 2 in a simple way that means: when the angles of the manipulators change, the center of gravity of the helicopter changes too. So we need enough torque for the main and tail rotors to make the helicopter fixed in its position in space. 

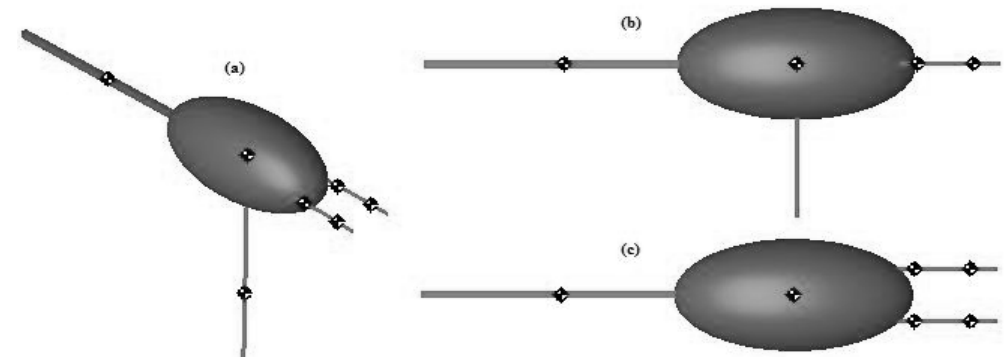

Figure 1. Helicopter model with manipulator, (a) isometric view (b) front view (c) top view
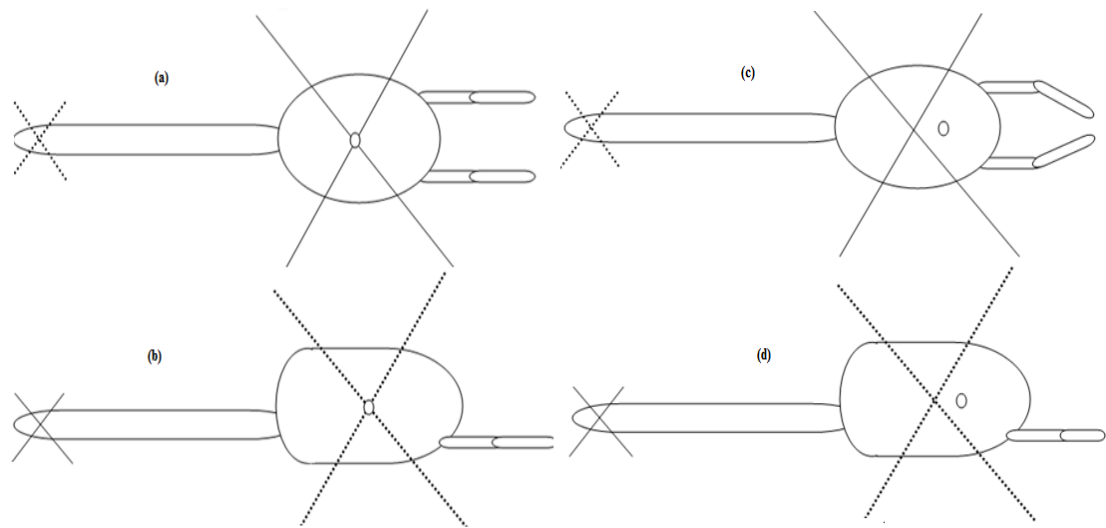

Figure 2. Changes of center of gravity (a) and (b) before manipulator movement, (c) and (d) after manipulator movement

\section{Simulation of System with SimMechanics}

In order to have a visual view of the system, we need to use a good toolbox to design the system. SimMechanics of Matlab software is a complete toolbox that we can use in order to have the helicopter and its manipulators simulated.

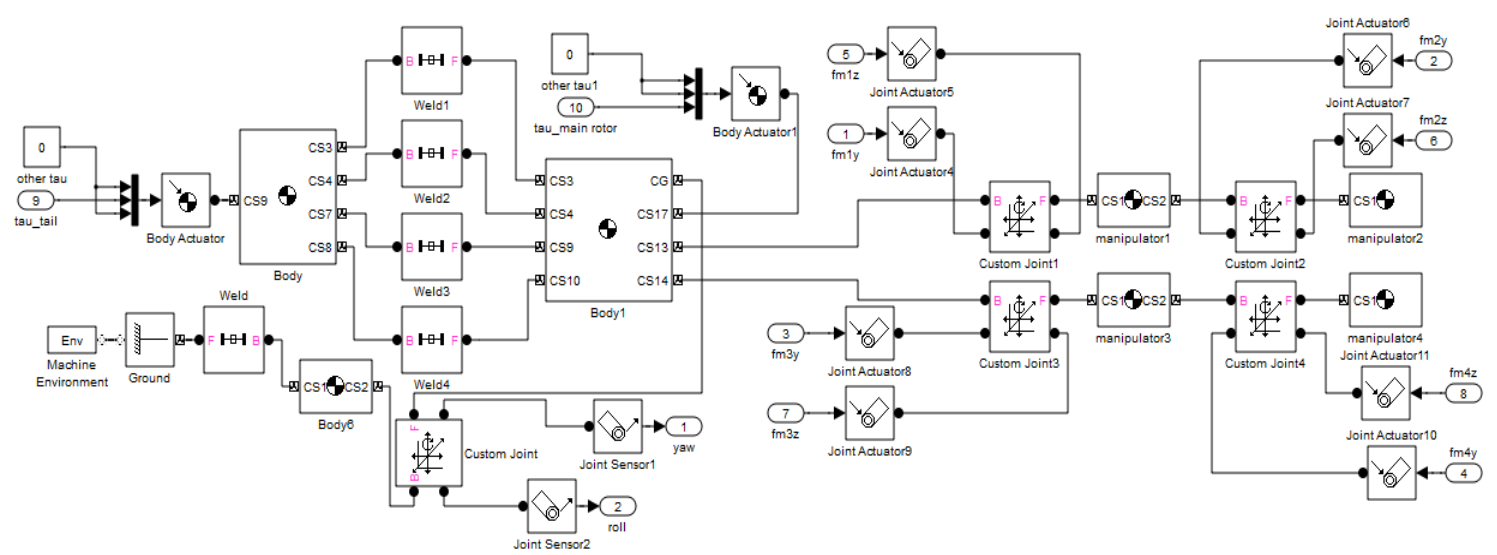

Figure 3. Schematic of the helicopter model and its manipulators in SimMechanics

SimMechanics is based on simulink, which is the research and analysis environment of the controller and the object system in a cross-cutting/interdisciplinary [8]. Multi-body daynamic mechanical systems can be analyze and modeled by SimMechanics and all works such as control would be completed in the simulink envirement. This toolbox provides a plenty number of corresponding real system components, such as: bodies, joints, constraints, coordinate 
systems, actuators and sensors. Complex mechanical system can be created by these modules in order to analyze them. In this paper, the toolbox has been used to analyze the helicopter model with articulated manipulators. Figure 3 illustrated the schematic of Helicopter model designed in SimMechanics.

\section{Fuzzy Logic Controller}

The FLC system, first proposed by Zadeh [17]. The system is also known as fuzzy control system or fuzzy inference system or approximate reasoning or expert system. The general framework of this system is shown in Figure 4. According to this framework, we designed the close loop system in Matlab which is illustrated in Figure 5.

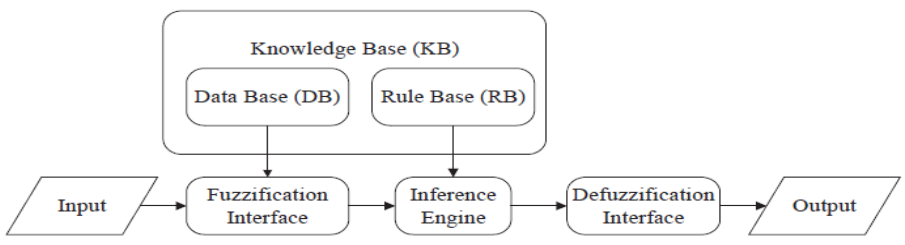

Figure 4. Framework of fuzzy logic controller

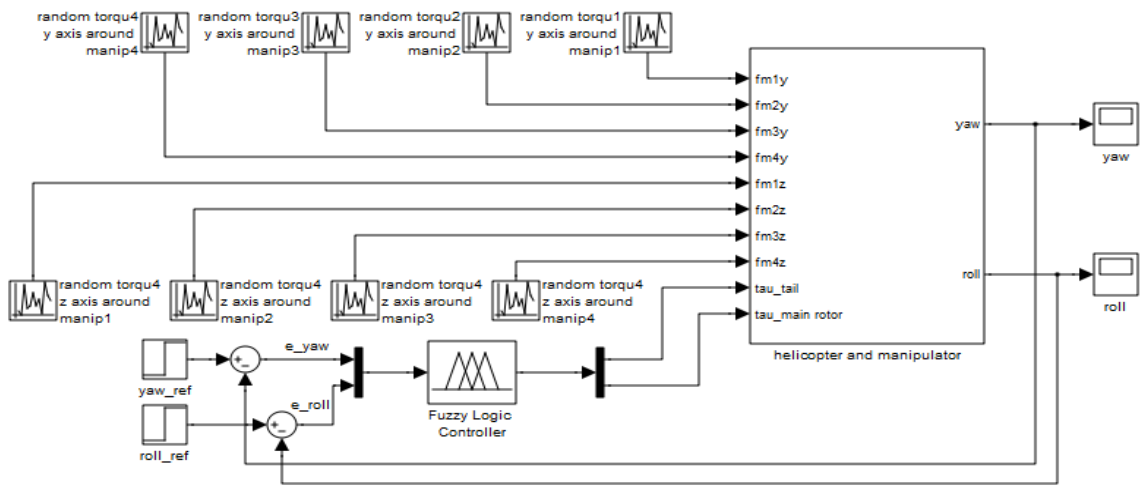

Figure 5. Schematic of fuzzy control system in Matlab Simulink
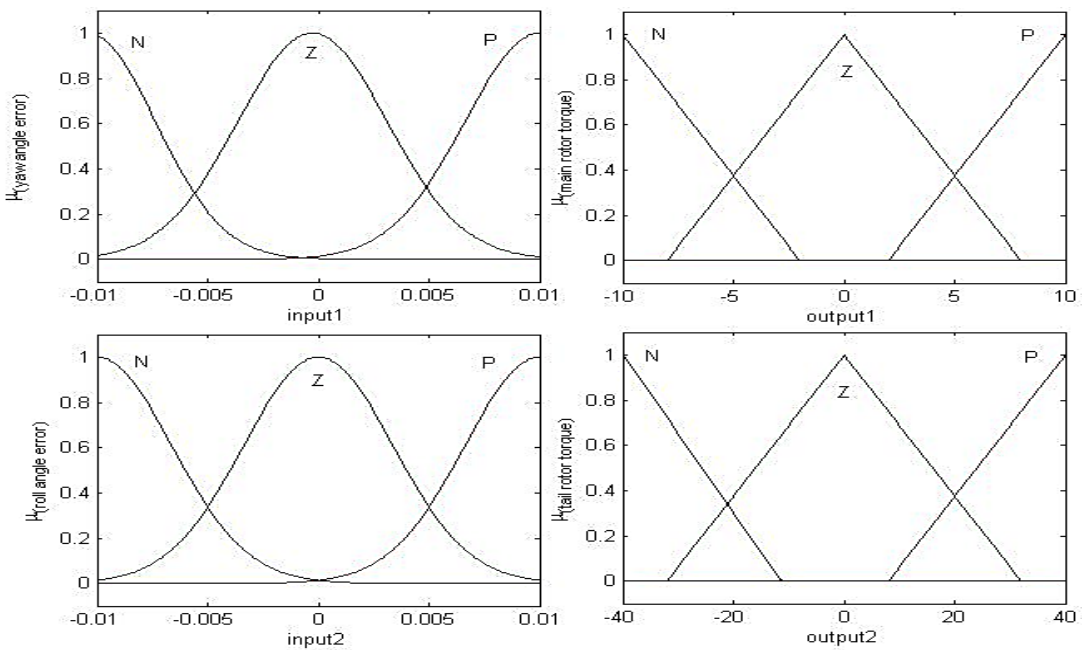

Figure 6. Membership functions of inputs and outputs 
In this paper, for the fuzzification process, the Gaussian membership functions and the Triangular membership functions are devoted to the inputs and outputs respectively with the universe of discourse as follows:

$$
e=[-0.01,0.01] \mathrm{deg}, \tau_{\text {yaw }}=[-10,10] \mathrm{N} . \mathrm{m}, \quad \tau_{\text {roll }}=[-40,40] \mathrm{N} . \mathrm{m}
$$

The defuzzification technique used in this study was Center of Gravity approach. Membership functions of inputs and outputs are illustrated in Figure 6.

The simple rule base used in the controller designing is given as follows:

if (input 1 is $N$ ) then (output 1 is $P$ )

if (input 1 is $P$ )then (output 1 is $N$ )

if (input 1 is Z) then (output 1 is Z)

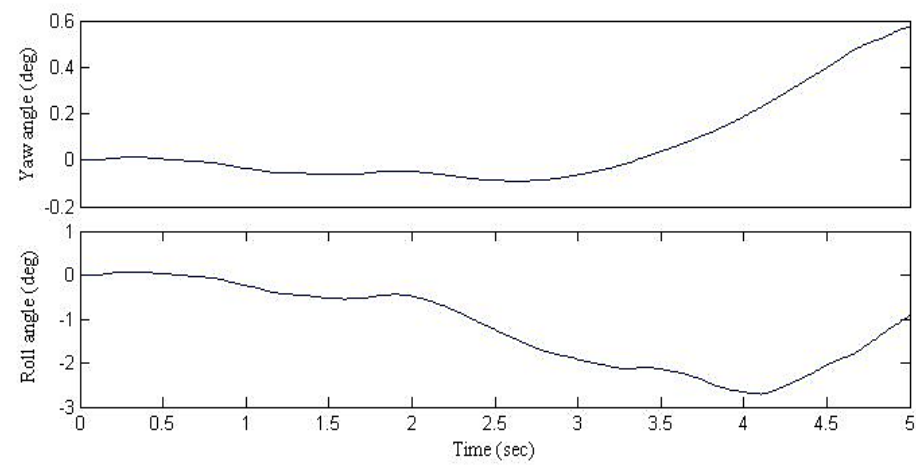

Figure 7. Changes of angles before adding a fuzzy controller

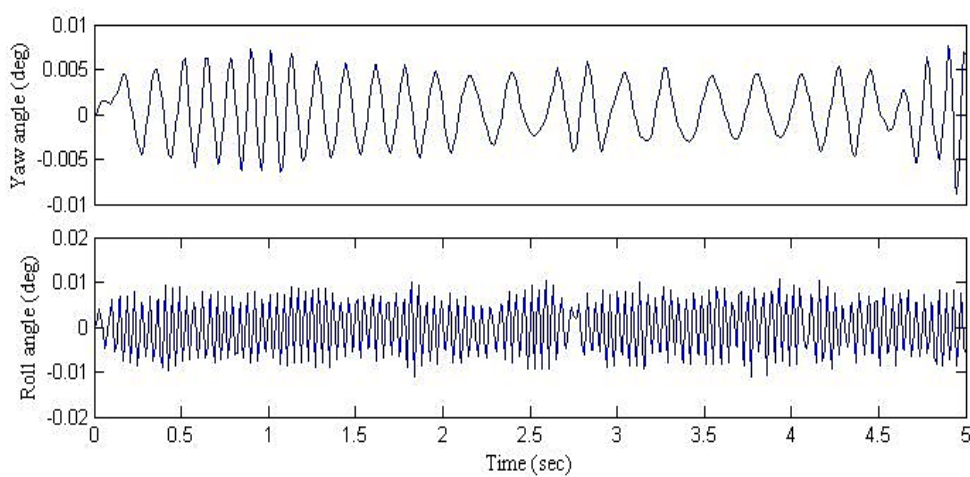

Figure 7. Changes of angles after adding a fuzzy controller

if (input2 is $N$ )then (output2 is $P$ )

if (input2 is $P$ ) then (output2 is $N$ )

if (input2 is Z) then (output2 is Z)

if (input1 is $N$ ) and (input2 is $N$ ) then (output1 is $\mathrm{P}$ ) and (output2 is P)

if (input 1 is $P$ ) and (input2 is $P$ ) then (output1 is $N$ ) and (output2 is $N$ )

if (input1 is Z) and (input2 is Z) then (output1 is Z) and (output2 is Z)

In this paper, our goal was to make the yaw and roll angle of the helicopter at zero(deg) and according to the designed controller we got a good response that is illustrated in Figure 7 and 8. Figure 7 shows the open loop operation of the system and Figure 8 shows the close loop system by adding a fuzzy controller. These results confirm that the fuzzy logic controller is suitable for controlling this system. 


\section{Conclusion}

In this paper, a new mechanical system has been introduced for modeling and applying different kinds of controllers on it. An attempt to control the yaw and roll angles of an arbitrary simulated helicopter includes manipulator by fuzzy logic has been proposed. From the simulation results, it has been shown that the fuzzy logic controller can make a suitable regulation in this system.

\section{References}

[1] Phillips C, Karr CL, Walker G. Helicopter flight control with fuzzy logic and genetic algorithms. Engineering Applications of Artificial Intelligence. 1996; 9(2): 175-184.

[2] Marconi L, Naldi R. Robust full degree of freedom tracking control of a helicopter. Automatica. 2007; 43(11): 1909-1920.

[3] Liu C, Chen WH, Andrews J. Tracking control of small-scale helicopters using explicit nonlinear MPC augmented with disturbance observers. Control Engineering Practice. 2012; 20(3): 258-268.

[4] Lee CT, Tsai CC. Nonlinear adaptive aggressive control using recurrent neural networks for a small scale helicopter. Mechatronics. 2010; 20(4): 474-484.

[5] Sanchez EN, Becerra HM, Velez CM. Combining fuzzy, PID and regulation control for an autonomous mini-helicopter. Information Sciences. 2007; 177(10): 1999-2022.

[6] Hernandez-Gonzalez M, Alanis AY, Hernandez-Vargas EA. Decentralized discrete-time neural control for a Quanser 2-DOF helicopter. Applied Soft Computing. 2012; 12( 8): 2462-2469.

[7] Furuta K, Ohyama Y, Yamano O. Dynamics of RC helicopter and control. Mathematics and Computers in Simulation. 1984; 26(2): 148-159.

[8] Zheng-wen LI, Guo-liang Zhang, Wei-ping Zhang, Bin JIN. A Simulation Platform Design of Humanoid Robot Based on SimMechanics and VRML. Procedia Engineering. 2011; (15): 215-219.

[9] Linear Control system Analysis and Design: Conventional and Modern. $4^{\text {th }}$ Edition: McGraw-Hill Companies.1995.

[10] ]van der Wal AJ. Application of fuzzy logic control in industry. Fuzzy Sets and Systems. 1995; 74(1): 33-41.

[11] Bortolet P, Merlet E, Boverie S. Fuzzy modeling and control of an engine air inlet with exhaust gas recirculation. Control Engineering Practice. 1999; 7(10): 1269-1277.

[12] Emami MR, Goldenberg AA, Türksen IB. Fuzzy-logic control of dynamic systems: from modeling to design. Engineering Applications of Artificial Intelligence. 2000; 13(1): 47-69.

[13] Tunstel E, Hockemeier S, Jamshidi M. Fuzzy control of a hovercraft platform. Engineering Applications of Artificial Intelligence. 1994; 7(5): 513-519.

[14] McLean D, Matsuda H. Helicopter station-keeping: comparing LQR, fuzzy-logic and neural-net controllers. Engineering Applications of Artificial Intelligence. 1998; 11(3): 411-418.

[15] Babuška R, Verbruggen HB. An overview of fuzzy modeling for control. Control Engineering Practice.1996; 4(11): 1593-1606.

[16] Fraichard Th, Garnier Ph. Fuzzy control to drive car-like vehicles. Robotics and Autonomous Systems. 2001; 34(1): 1-22.

[17] Zadeh L. Outline of a new approach to the analysis of complex systems and decision processes. IEEE Trans. Systems, Man Cybernet SMC-3. 1973; 28-44. 